\title{
Inhibition of Pancreatic Carcinoma Growth Through Enhancing (0-3 Epoxy Polyunsaturated Fatty Acid Profile by Inhibition of Soluble Epoxide Hydrolase
}

\author{
RONG XIA ${ }^{1 *}$, LEYU SUN $^{1 *}$, JIE LIAO $^{1 *}$, HAONAN LI $^{1}$, XIAOMING YOU $^{1}$, DANDAN XU ${ }^{1}$, JUN YANG $^{2}$, \\ SUNG HEE HWANG ${ }^{2}$, RYAN D. JONES ${ }^{1}$, BRUCE HAMMOCK ${ }^{2}$ and GUANG-YU YANG ${ }^{1}$ \\ ${ }^{I}$ Department of Pathology, Northwestern University, Feinberg School of Medicine, Chicago, IL, U.S.A.; \\ ${ }^{2}$ Department of Entomology, University of California, Davis, CA, U.S.A.
}

\begin{abstract}
Background/Aim: Cytochrome P450 epoxygenase is a major enzyme involved in the metabolism of $\omega-3$ polyunsaturated fatty acids (PUFAs) to produce biologically active $\omega$-3 epoxy fatty acids ( $\omega-3$ epoxides). In general, all epoxy PUFAs including $\omega-3$ epoxides are quickly metabolized/inactivated by soluble epoxide hydrolase ( $s E H$ ) to form diol products. The aims of this study were to determine the effect and mechanism of fat-1 transgene, and $\omega-3$ PUFA combined with sEH gene knockout or inhibitor on inhibiting pancreatic cancer and the related mechanisms involved. Materials and Methods: PK03-mutant Kras ${ }^{G 12 D}$ murine pancreatic carcinoma cells were inoculated into mouse models including fat-1, sEH ${ }^{-1-}$ and $\mathrm{C} 57 \mathrm{BL} / 6 \mathrm{~J}$ mice. The mice were fed with AIN-76A diet with or without $\omega-3$ PUFA supplementation or treated with $s E H$ inhibitor. In addition to tumor growth (tumor size and weight), cell proliferation, mutant Krasmediated signaling, inflammatory reaction and angiogenesis were analyzed immunohisto-chemically and by western blot assay. $\omega-3$ PUFA metabolism, particularly focusing on $\omega-3$ epoxy fatty acids ( $\omega$-3 epoxides), was measured using a liquid chromatography with tandem mass spectrometry (LC-MS/MS) approach. Results: Significant decreases of weight and size of the PKO3 pancreatic carcinoma were observed in the fat-1 transgenic mice treated with $\mathrm{SEH}$ inhibitor compared to those of C57BL/6J control mice fed with AIN-76A diet (weight: $0.28 \pm 0.04 \mathrm{~g}$ vs. $0.58 \pm 0.06 \mathrm{~g}$; size: $187.0 \pm 17.5 \mathrm{~mm}^{3} \mathrm{vs}$.
\end{abstract}

*These Authors contributed equally to this study.

Correspondence to: Guang-Yu Yang, MD, Ph.D., Department of Pathology, Northwestern University, Feinberg School of Medicine, 303 E. Chicago Ave, Ward 4-115, Chicago, IL 60611, U.S.A. Tel: +1 3125030645, Fax: +1 3125030647, e-mail: g-yang@northwestern.edu

Key Words: Polyunsaturated fatty acid, epoxy fatty acid, fat-1, soluble epoxide hydrolase, pancreatic cancer. $\left.519.3 \pm 60.6 \mathrm{~mm}^{3}\right)$. In a separate experiment, $\mathrm{sEH} \mathrm{H}^{-1-}$ mice fed $\omega$-3 PUFA supplement and C57BL/6J mice treated with $s E H$ inhibitor and fed $\omega-3$ PUFA supplement exhibited a significant reduction in the weight and size of the pancreatic carcinoma compared to C57BL/6J control mice (weight: $0.26 \pm .26 \mathrm{~g}$ and $0.39 \pm .39 \mathrm{~g}$ vs. $0.69 \pm 0.11 \mathrm{~g}$, respectively; size: $274.2 \pm 36.2 \mathrm{~mm}^{3}$ and $296.4 \pm 99.8 \mathrm{~mm}^{3} \mathrm{vs} .612 .6 \pm 117.8 \mathrm{~mm}^{3}$, respectively). Moreover, compared to the pancreatic tumors in $C 57 \mathrm{BL} / 6 \mathrm{~J}$ control mice, the tumors in fat-1 transgenic mice treated with sEH inhibitor showed a significant less inflammatory cell

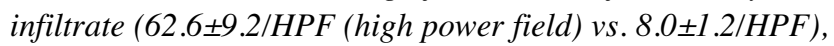
tumor cell proliferation $(48.5 \pm 1.7 \%$ vs. $16.5 \pm 1.6 \%)$, and angiogenesis (micro-vessel density (MVD): $35.0 \pm 1.0 \mathrm{vs.}$ $11.1 \pm 0.5)$ immunohistochemically, as well as significantly increased caspase-3 labeled apoptosis $(0.44 \pm 0.06 \%$ vs. $0.69 \pm 0.06 \%$, respectively). Using western blot approach, significant inhibition of mutant Kras-activated signals including phosphorylated Serine/threonine kinases (cRAF), Mitogen-activated protein kinase kinase (MEK), and extracellular signal-regulated kinase (ERK) were identified in pancreatic carcinoma of fat-1 transgenic mice treated with sEH inhibitor. Eicosanoic acid metabolic profiling of the serum specimens detected a significant increase of the ratios of epoxides to dihydroxy fatty acid (DiHDPE) for docosahexaenoic acid (DHA) and eicosapentaenoic acid (EPA), and epoxides/dihydroxy octadecenoic acid (DiHOME) for arachidonic acid (ARA) and linoleic acid (LA), as well as a significant increase of epoxy metabolites of DHA, EPA, ARA and LA in fat-1 transgenic mice treated with a sEH inhibitor. Conclusion: $\omega-3$ epoxy products from $\omega-3$ PUFA metabolism play a crucial role in inhibiting pancreatic cancer growth, and use of $\omega-3$ PUFAs combined with sEH inhibition is a strategy with high potential for pancreatic cancer treatment and prevention.

$\omega-3$ Polyunsaturated fatty acids ( $\omega-3$ PUFAs) are the essential long-chain fatty acids that have numerous beneficial 
effects for the most common metabolic diseases including cardiovascular diseases and cancer. Three natural $\omega$-3 PUFAs in food are $\alpha$-linolenic acid (ALA) mainly present in plant/nut oils, and eicosapentaenoic acid (EPA) and docosahexaenoic acid (DHA) both mainly present in fish oils. Anti-inflammatory and anti-carcinogenic effects of $\omega-3$ PUFAs have been well identified or documented, but the mechanisms involved remain to be further investigated. Cyclo-oxygenase (COX), lipoxygenase and cytochrome P450 are three major enzymes involved in PUFA metabolism. Recent studies indicate that $\omega-3$ PUFAs are mainly metabolized by cytochrome $P 450$ epoxygenases to produce $\omega-3$ epoxy fatty acid metabolites (called $\omega-3$ epoxides), such as 17,18-epoxyeicosatetraenoic acid derived from EPA and 19,20-epoxydocosapentaenoic acid from DHA (1-3). COX and lipoxygenase pathways play a minimal role in $\omega$-3 PUFA metabolism (4-6). Endogenous $\omega-3$ epoxides have highly polarized oxygen-carbon bonds, making them crucial signaling molecules. Several studies have indicated the importance of $\omega-3$ epoxides as highly potent metabolites against inflammation and carcinogenenesis, particularly via targeting inflammatory signals (7-10). Since soluble epoxide hydrolase $(\mathrm{sEH})$ as a proinflammatory enzyme inactivates these $\omega-3$ epoxides through metabolizing them to the less active diol products, targeting $\mathrm{sEH}$ should be a potential strategy for stabilizing these $\omega-3$ epoxides and enhancing their beneficial actions (11-14).

sEH has very restricted substrates that are endogenous $\omega-3$ and $\omega-6$ lipid epoxides, but not toxic or mutagenic xenobiotics (14). Stabilizing epoxide metabolites by $\mathrm{sEH}$ inhibition appears crucial in action against inflammation and tumor growth. A potent sEH inhibitor, trans-4-[4-(3adamantan-1-ylureido)cyclohexyloxy]benzoic acid ( $t$-AUCB), a transition state mimic inhibitor, was designed and optimized mainly based on the catalytic mechanism of $\mathrm{sEH}$, with the inhibitor constant $\mathrm{Ki}$ in the nano- to picomolar ranges $(15,16)$. Our previous studies showed a significant chemopreventive effect of $t$-AUCB on colitis-induced carcinogenesis in mice; and such effect was comparable to that of sEH gene (EPHX2 epoxide hydrolase 2 gene) knockout in mice (17). $\omega-3$ and $\omega-$ 6 PUFA are two major long chain fatty acids in the diet (4). Epidemiological and experimental studies provide evidence that $\omega$-3 PUFAs are beneficial for reducing the risk of cancer, whereas $\omega-6$ PUFAs have opposite effects (18), and a high ratio of dietary $\omega-6 / \omega-3$ PUFAs increases the risk of cancer (19) and obesity-associated inflammation and insulin resistance (20). The relative contribution of $\omega-3$ epoxides as key metabolites of $\omega-3$ PUFAs in anticancer/antiinflammatory activity are not well studied.

Caenorhabditis elegans fat 1 gene encodes $\omega$-3 fatty acid desaturase, and heterozygous fat -1 transgenic mice have an abundance of $\omega-3$ PUFAs and high $\omega-3 / \omega-6$ PUFA ratio in their organs and tissues from their embryonic stage throughout their lives obviously via endogenously converting $\omega-6$ PUFAs to $\omega-3$ PUFAs by the transgenic $\omega-3$ fatty acid desaturase (21). The heterozygous fat- 1 transgenic mouse is a wellcontrolled experimental model of $\omega-3$ PUFAs that is significant for studying biology and potential beneficial effects of $\omega-3$ fatty acids and their metabolites such as epoxides. Heterozygous fat- 1 transgenic mice are identified as having no metabolic abnormalities and are phenotypically normal and healthy through many generations of transgenic mouse lines. But homozygosity for fat- 1 transgene is lethal, indicating that a proper balance of the $\omega-3 / \omega-6$ PUFA ratio is crucial.

Pancreatic ductal adenocarcinoma (PDAC) is the fourth leading cause of cancer-related death in the United States (22). Prevention of highly lethal pancreatic cancer, particularly by developing efficient and safer agents, is of vital significance $(23,24)$. Pancreatic cancer follows a multiple stage progression model from pancreatic intraepithelial neoplasia to invasive adenocarcinoma with the accumulation of genetic alterations. In particular, oncogenic mutation of Kirsten RAS oncogene (25) is an early event and the most common mutation, being found in $>90 \%$ of PDACs $(11,26)$. At least four critical signal transduction pathways are regulated by active mutant KRAS, including the Serine/threonine kinases (RAF)- Mitogen-activated protein kinase kinase (MEK)-extracellular signal-regulated kinase (ERK) pathway, which is not only necessary for cell proliferation and apoptosis, but is also essential for tumor initiation and maintenance (27). It has been shown that targeting mutant KRAS and the activated RAF-MEK-ERK cascade induces apoptosis (28), and is significant for prevention and therapy of this lethal malignancy.

The PK03 mouse pancreatic ductal carcinoma cell model is well established in our laboratory, and was derived from the pancreas of a 6-month-old PdxI-Cre;LSL-Kras G12D mouse $(24,29)$. This PK03 cell model was demonstrated to i) be of pancreatic ductal epithelial origin as evidenced by the expression of cytokeratin 19 and E-cadherin, but not amylase; ii) display a neoplastic feature in vitro (clonal formation) and in vivo (tumor formation when inoculated into C57BL/6J mice); and iii) have an activated transgenic mutant $\mathrm{Kras}^{\mathrm{G} 12 \mathrm{D}}$ gene. In the present study, the synergistic effects of fat-1 transgene or a $\omega-3$ PUFA-enriched diet combined with $\mathrm{sEH}$ inhibitor $t$-AUCB or sEH gene knockout on pancreatic carcinoma growth were determined using our murine PK03 pancreatic ductal carcinoma cell model with the following animal experiments: i) fat- 1 transgenic mice treated with $\mathrm{sEH}$ inhibitor $t$-AUCB; ii) $s E H^{-1-}$ mice fed with $\omega-3$ PUFAenriched AIN-76A diet; and iii) C57BL/6J mice treated with $t$-AUCB and fed with $\omega-3$ PUFA-AIN-76A diet. Eicosanoic acid metabolic/lipidomic profiling was analyzed by a liquid chromatography with tandem mass spectrometry (LC-MS/MS) method. Using western blot and immunohistochemical staining approaches, we analyzed the tumor growth, KRAS 
downstream signaling, intratumoral angiogenesis and inflammation in the tissues and tumor obtained from these animal experiments.

\section{Materials and Methods}

PKO3 cells. An in vitro-cultured mouse pancreatic carcinoma cell model/line (PK03) established in our laboratory was used for this study as previously described $(24,29)$. Cells were cultured and grown in a monolayer in Dulbecco's modified Eagle's medium (DMEM) with $10 \%$ fetal bovine serum, $0.1 \%$ gentamicin and $0.1 \%$ insulin, and maintained at $37^{\circ} \mathrm{C}$ with $5 \% \mathrm{CO}_{2}$. Cell proliferation and viability were measured for viable and non-viable cells using a trypan blue staining.

Animal care, breeding and genotyping. All animal experiments were approved by the Institutional Animal Care and Use Committee at Northwestern University (NU2011-1389). C57BL/6J mice were purchased from Jackson Laboratory (Bar Harbor, ME, USA). $s E H^{-/}$ mice in a C57BL/6J background were obtained from Dr. Hammock, the University of California, Davis, CA, USA. fat- 1 transgenic mice in the C57BL/6J background were a gift from Dr. Jing X. Kang (Department of Medicine, Massachusetts General Hospital and Harvard Medical School, Boston, MA, USA).

Safflower oil consists primarily of triglycerides of linoleic acid (LA; 18:2n6) and has very low levels of $\omega-3$ PUFA and no DHA (30). Modified AIN-76A Purified Rodent Diet containing 10\% SFO (Dyets Inc., Bethlehem, PA, USA) was the sole diet fed for all fat- 1 transgenic mice, including fat- 1 offspring weaned at 3 weeks of age. $\omega$-3 PUFA-enriched diet was made by adding $1.5 \%$ fish oil of the total 5\% fat (corn oil-based) in AIN-76A diet. All mice were housed in micro-isolator cages in the animal facilities at the Center for Comparative Medicine of Northwestern University. Genotyping of fat-1 gene was performed following the literature method $(19,22)$.

Subcutaneous implantation of pancreatic PKO3 cancer cells in mice, tumor growth and tissue specimen processing. PK03 cells $\left(3 \times 10^{6}\right.$ cells per $100 \mu \mathrm{l}$ per inoculation site per mouse) were injected subcutaneously into both hind limbs of fat $-1, s E H^{-1-}$ and $\mathrm{C} 57 \mathrm{BL} / 6 \mathrm{~J}$ mice (7-8 weeks old, 18-22 g, $\mathrm{n}=12$ per group). SFO AIN-76A diet was given to fat-1 transgenic mice. Either AIN-76A or $\omega$-3 PUFAAIN-76A diet was given to $s E H^{-1-}$ and C57BL/6J mice. sEH inhibitor $t$-AUCB (provided by Dr. Hammock) was administered to fat-1 transgenic mice and $\mathrm{C} 57 \mathrm{BL} / 6 \mathrm{~J}$ mice in a drinking water at a concentration of $95 \mathrm{mg} / \mathrm{l}(10 \mathrm{mg} / \mathrm{kg} / \mathrm{day})$ starting on the day of implantation and continuously to the end of the experiment. PEG400 was used as a solvent for $t$-AUCB and PEG-400 water as vehicle control were given to the corresponding control groups.

Food and water consumption, and body weight were monitored weekly. The tumor development was monitored daily. Mice were sacrificed after 4 weeks when tumor size reached $1 \mathrm{~cm}$ in diameter in any group. Blood and plasma were collected via the heart following euthanasia by $\mathrm{CO}_{2}$ and were stored at $-80^{\circ} \mathrm{C}$ until analysis. Tumors were dissected and measured for size (length and width) and weight. Tumor volume was determined using the equation $\mathrm{V}=4 / 3 \pi r^{3}$, where $r$ was the average tumor radius obtained from three measurements of diameter. Half of each tumor was then fixed in $10 \%$ buffered formalin and processed for paraffin sections for histopathological and immunohistochemical analyses. The other half was stored at $-80^{\circ} \mathrm{C}$ until biochemical analyses.
Immunohistochemical (IHC) staining. Following our previously described method and using the Avidin-Biotin complex (ABC) approach (31), IHC staining of paraffin-embedded tumor sections was performed. The antibodies and reagents used were antimyeloperoxidase (MPO; Rabbit polyclonal antibody, 1:100 dilution; Abcam, Cambridge, MA, USA), anti-Ki67 (Rabbit polyclonal antibody, 1:100 dilution; Vector Laboratories Inc., Burlingame, CA, USA), biotinyted anti-rabbit IgG secondary antibody (1:200; Vector Laboratories Inc.), ABC (Vector Laboratories Inc.), and diaminobenzidine (Sigma-Aldrich, St. Louis, MO). The number of MPO-positive inflammatory cells per high-power field (HPF; 40x objective lens) was counted for each specimen in at least 10 fields per tumor slide and expressed as a percentage. Cell proliferation as the proliferative index was analyzed as the percentage of Ki67labeled cells out of the total number of cells counted. New vessel formation (angiogenesis) was analyzed immunohistochemically using antibody to CD34 (Rabbit monoclonal antibody, 1:100 dilution, Abcam, Cambridge, MA, USA). CD34-labeled microvessels were counted and expressed as microvessel density (MVD; stained vessel per HPF) for at least 10 fields per tumor slide.

Protein extraction and western blotting. Following our previously described methods $(32,33)$, freshly harvested tumor tissues were extracted for cell lysates using ice-cold RIPA lysis buffer (Santa Cruz Biotechnology, Santa Cruz, CA, USA) and total protein concentrations were measured using the Bradford reagent (Thermo Scientific, Chicago, IL, USA). The tissue lysate (30 $\mu \mathrm{g}$ protein/lane) was separated by $10 \%$ sodium dodecyl sulfate-polyacrylamide gel electrophoresis and transferred onto a polyvinylidene fluoride membrane. The specific targeting signals were probed by primary antibodies against phospho (p)-ERK, ERK, p-MEK, MEK, p-c-RAF, and c-RAF, as well as E-cadherin (Rabbit monoclonal antibody, 1:1,000 dilution, Abcam, Cambridge, MA, USA) and horseradish peroxidase-conjugated secondary antibody, followed by the SuperSignal West Pico kit (Pierce, Appleton, WI, USA). All antibodies were purchased from Cell Signaling Technology, Danvers, MA, USA. The band densities on photographic films were qualitatively analyzed using Photoshop software (Adobe, San Jose, CA, USA) and further normalized to the internal control band of $\beta$-actin.

Metabolic profile-lipidomics of oxylipins analyzed by a LC-MS/MS method. LC-MS/MS analysis of oxylipins to produce a lipidomic profile was performed based on our previous publication for freshly collected serum specimens (23). Over 120 oxylipins including cyclooxygenase, lipoxygenase and cytochrome P450-mediated PUFA metabolites were analyzed. Calibration curves were generated using $10 \mu \mathrm{l}$ injections of seven standards containing each analyte, internal standard I, and internal standard II for quantification purpose.

Statistical analysis. Each quantitative parameter analyzed was expressed as the mean \pm SEM. Student $t$-test or two-way ANOVA was use for the analysis of statistical significance, and differences were considered statistically significant when $p<0.05$.

\section{Results}

fat-1 transgene or $\omega-3$ PUFAs supplementation together with either sEH inhibition or knockout suppresses pancreatic carcinoma cell growth in mice. First, fat-1 transgenic mice as a powerful model of $\omega-3$ PUFAs were used in combination 
with sEH inhibitor $t$-AUCB to determine the effect of $\omega-3$ epoxides on PK03 pancreatic ductal carcinoma cell growth. 1) In fat-1 transgenic mice treated with $\mathrm{sEH}$ inhibitor compared to no sEH inhibitor control, the weight and size of PK03 pancreatic carcinoma were significantly reduced (both $p<0.05$ by two-way ANOVA); but in C57BL/6J mice treated with sEH inhibitor compared to no sEH inhibitor control, there was no statistically significant effect on tumor weight and size ( $p>0.05)$, as seen in Figure 1A-C. 2) For comparison between fat-1 transgenic mice and C57BL/6J mice treated with and without sEH inhibitor, similarly as above, fat-1 transgenic mice treated with $\mathrm{sEH}$ inhibitor made more significant difference in both the weight and size of the tumor (two-way ANOVA, $p<0.05$ ). Histopathological analysis revealed that the PK03 pancreatic carcinoma displayed more glandular formation/well-differentiated tumor in wild-type C57BL/6J mice and in fat-1 transgenic mice not treated with sEH inhibitor, and more stromal hyalinization in fat- 1 transgenic mice treated with sEH inhibitor (Figure 1D).

To determine the effect of dietary $\omega$-3 PUFA supplementation together with sEH gene knockout or sEH inhibition on PK03 pancreatic carcinoma growth, two additional experiments were performed using $s \mathrm{EH}^{-/}$mice and $\mathrm{C} 57 \mathrm{BL} / 6 \mathrm{~J}$ mice inoculated with PK03 cells. As seen in Figure 1E and F, PK03 pancreatic carcinoma growth as measured by tumor weight and size was significantly smaller in $s E H^{-1-}$ mice fed $\omega$-3 PUFA-enriched diet compared with $s \mathrm{EH}^{-1-}$ mice fed with control AIN-76A diet $(p<0.05)$. Similarly, C57BL/6J mice fed $\omega$-3 PUFA-enriched diet and treated with $t$-AUCB displayed significant smaller PK03 pancreatic carcinoma growth than that in C57BL/6J mice fed with control AIN-76A diet and treated with $t$-AUCB $(p<0.05)$ and in those without $t$-AUCB treatment $(p<0.05)$.

Histopathological analysis showed the PK03 carcinoma exhibited minimal degenerative changes with hyalinized stroma reaction in fat 1 transgenic mice treated with $t$-AUCB (Figure 1D), but not in the mice in other groups. No metastasis was identified in key organs, including liver, lung, adrenal, kidney and gastrointestinal tracts, from the murine PK03 tumors in any experimental groups.

In addition, body weight, food and water consumption were routinely monitored during the animal experiment. No difference was observed in the average body weight, or food and water consumption between the fat 1 transgenic mice, $s E H^{-}$ ${ }^{\prime}-$ and wild-type mice treated with or without $t$-AUCB treatment or fed with $\omega$-3 PUFA-enriched diet (data not shown).

Cell proliferation, apoptosis, inflammatory cell infiltration and angiogenesis. Cell proliferation, apoptosis, inflammatory cell reaction and angiogenesis in the PK03 carcinomas were analyzed immunohistochemically. As seen in Figure 2A, numerous Ki-67-labeled proliferating cells were identified in tumors from C57BL/6J mice, and markedly fewer Ki-67labeled proliferating cells were observed in the tumors from fat 1 transgenic mice, regardless of treatment with $t$-AUCB. The proliferative index (percentage of Ki-67-positive cells out of the total cells counted) was statistically significantly reduced in the tumors from fat- 1 transgenic mice treated with $t$-AUCB compared to those not with $t$-AUCB $(p<0.05)$. Compared to $\mathrm{C} 57 \mathrm{BL} / 6 \mathrm{~J}$ mice, statistically significant lower $\mathrm{Ki}-67-$ labeled cell proliferation $(p<0.05)$ was seen in tumors from fat- 1 transgenic mice, regardless of $t$-AUCB treatment, as seen in Figure 2A. Significant induction of cell apoptosis labeled by caspase- 3 immunohistochemically was also observed in fat- 1 transgenic mice compared to $\mathrm{C} 57 \mathrm{BL} / 6 \mathrm{~J}$ mice $(p<0.05)$ regardless of $t$-AUCB treatment, as seen in Figure 2B.

Active inflammatory cells, neutrophils, were analyzed immunohistochemically using antibody to MPO. Anti-MPOlabeled neutrophil infiltrates were frequently observed in the tumor adjacent to normal tissues and rare positively stained cells were seen within tumors. As seen in Figure 2C, significantly fewer MPO-labeled neutrophils were observed in wild-type C57BL/6J mice treated with $t$-AUCB $(p<0.05)$ compared to $\mathrm{C} 57 \mathrm{BL} / 6 \mathrm{~J}$ mice without $t$-AUCB treatment. Similarly, inhibition of neutrophil infiltrate was also observed in fat- 1 transgenic mice treated with $t$-AUCB compared to those without $t$-AUCB treatment (34). Regardless of $t$-AUCB treatment, compared to C57BL/6J mice, fat- 1 transgenic mice showed a significant reduction of MPO-labeled neutrophils (Figure 2C, $p<0.05$ ).

Angiogenesis was analyzed immunohistochemically using antibody to CD34. Compared to C57BL/6J mice, the MVD was significantly lower observed in fat- 1 transgenic mice $(p<0.05)$, as seen in Figure 2D. Although an increased MVD was observed in mice treated with $t$-AUCB, no statistical difference was observed between mice receiving $t$-AUCB treatment and vehicle control, neither in $\mathrm{C} 57 \mathrm{BL} / 6 \mathrm{~J}$ mice nor in fat- 1 transgenic mice.

Signaling of mutant KRAS was analyzed using western blot assay of proteins obtained from the tumor tissues. The levels of phosphorylated c-RAF, MEK1/2 and ERK1/2 appeared to be markedly reduced in the tumors from fat-1 transgenic mice treated with $t$-AUCB, compared to those in untreated fat 1 transgenic mice and $\mathrm{C} 57 \mathrm{BL} / 6 \mathrm{~J}$ mice treated with/without $t$-AUCB (Figure 3). Increased expression of Ecadherin was observed in tumors from fat- 1 transgenic mice compared to C57BL/6J mice (Figure 3).

All of these biomarkers were also analyzed in the tumors in $s E H^{-1-}$ mice fed $\omega-3$ PUFA-enriched diet and C57BL/6J mice fed $\omega-3$ PUFA-enriched diet and treated with $t$-AUCB. Similar results to those of fat- 1 transgenic mice treated with $t$-AUCB were observed (data not shown).

PUFA metabolic profiling. The plasma PUFA metabolic/ lipidomic profile was analyzed using a LC-MS/MS approach. For lipidomic profile of both $\omega-3$ and $\omega-6$ fatty 
A

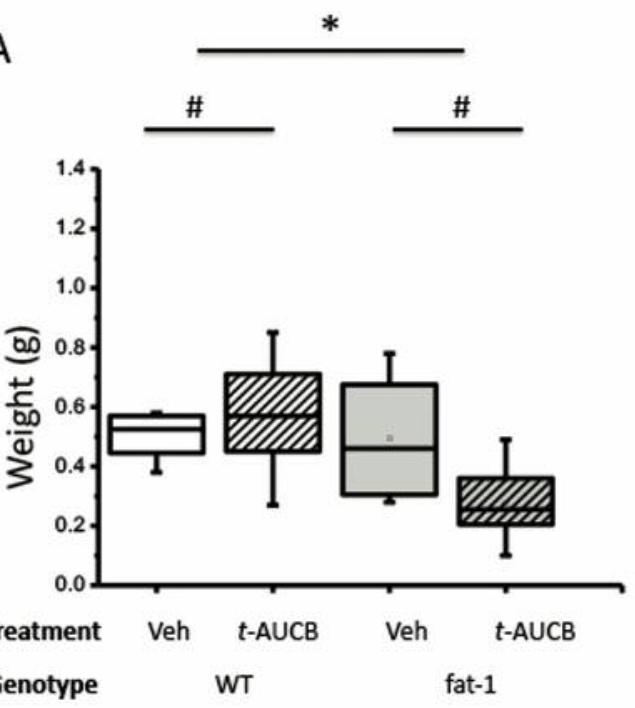

C

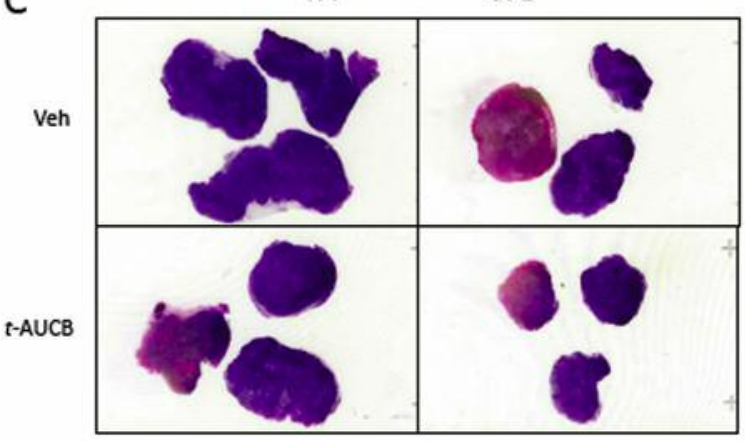

$\mathrm{E}$

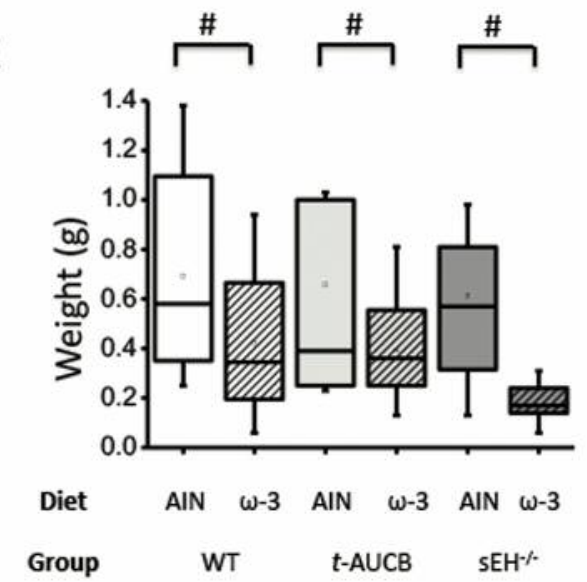

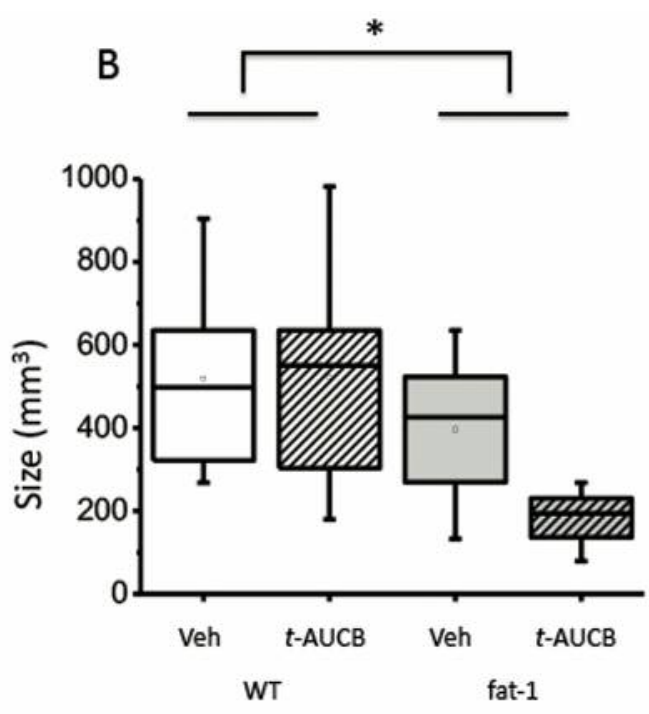

D
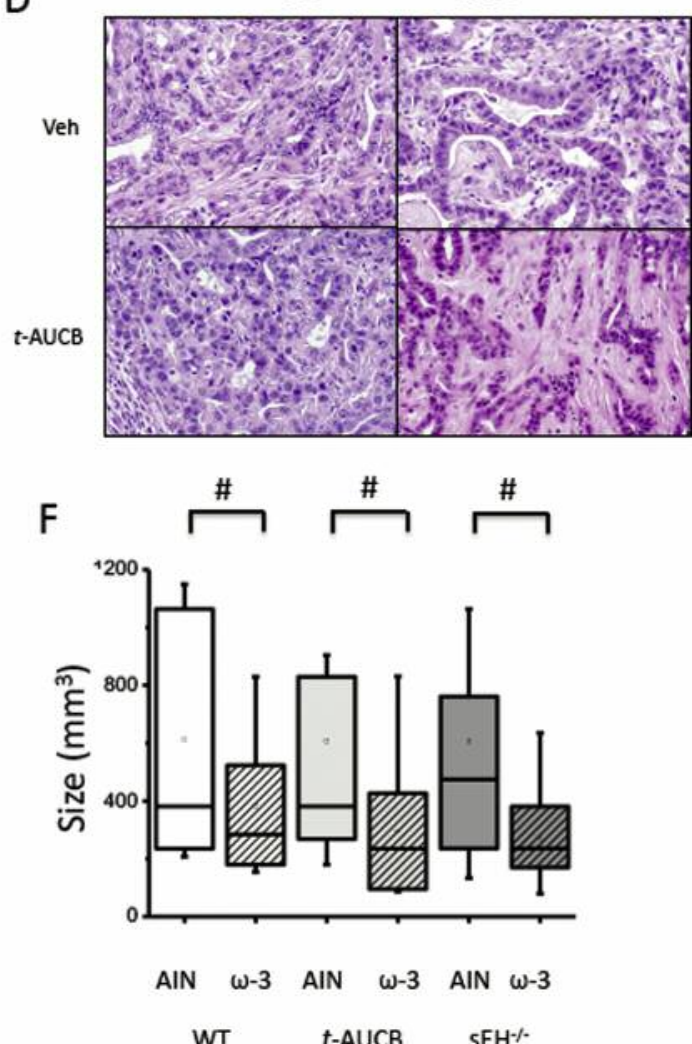

Figure 1. Growth and morphology of tumor of PK03 cells in the study groups. Trans-4-[4-(3-adamantan-1-ylureido)cyclohexyloxy]benzoic acid (t$A U C B$ ) dissolved in PEG-400 (also vehicle control) was administered to wild-type (WT) or fat-1 transgenic mice in drinking water at a concentration of $95 \mathrm{mg} / \mathrm{I}$ (final dose: $10 \mathrm{mg} / \mathrm{kg}$ body weight) starting on the day of implantation of PK03 cells and continuously to the end of the experiment ( $n=12$ for each group). PKO3 cells were also subcutaneously inoculated into the flanks of mice with soluble epoxide hydrolase (Ephx2) knockout $\left(s E H^{-l-}\right)$ and WT mice subsequently fed with either AIN-76A diet or 1.5\% fish oil-supplemented AIN-76A diet. Weight (A) and size (B) of the PK03 tumor in fat-1 transgenic mice and WT C57 BL/6J mice. Macro- $(C)$ and microscopic (D) images of the tumors from PK03 cells in WT and fat-1 transgenic mice. Weight $(E)$ and size $(F)$ of the PK03 tumor in WT mice treated with and without trans-4-[4-(3-adamantan-1-ylureido) cyclohexyloxy]benzoic acid ( $t$-AUCB), and in $s E H^{-1-}$ mice are illustrated in box plots. Each box represents the 25th to 75th percentiles. Squares inside the box represent the mean. Line inside the box indicate the median. Bars represent the SD. Means were significantly different between *groups with different genotype, and " groups receiving different treatments (two-way ANOVA, $p<0.05$ ). 

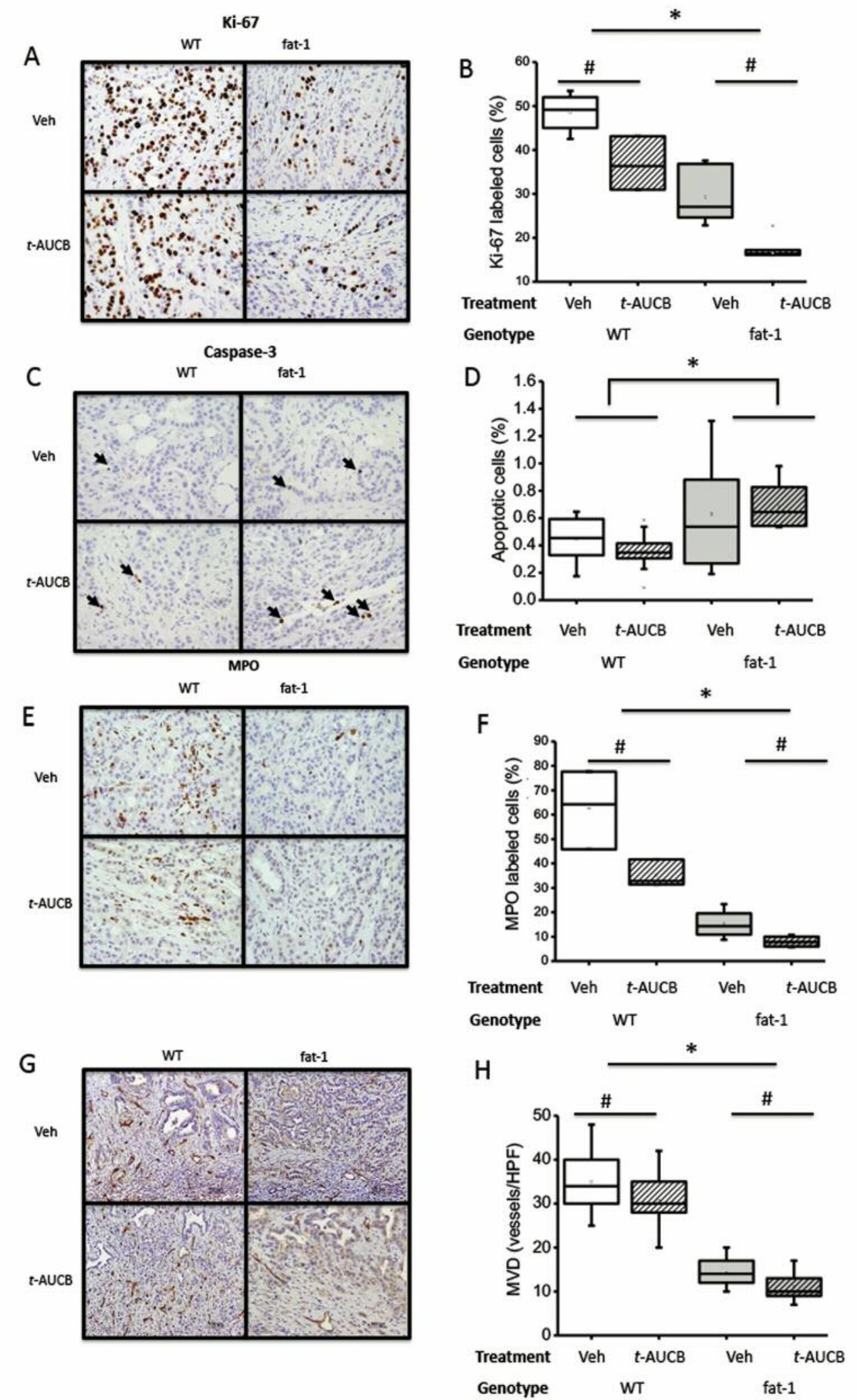

Figure 2. Immunohistochemistry (left panel) and quantification (right panel) of Ki-67 proliferative index (A), caspase-3-labeled apoptosis (B), myeloperoxidase (MPO)-labeled neutrophils $(C)$, and CD34-labeled microvessel density $(M V D)(D)$ in PK03 cell tumors. Means were significantly different between * groups with different genotype, and \#groups receiving different treatments (two-way ANOVA, $p<0.05$ ). 


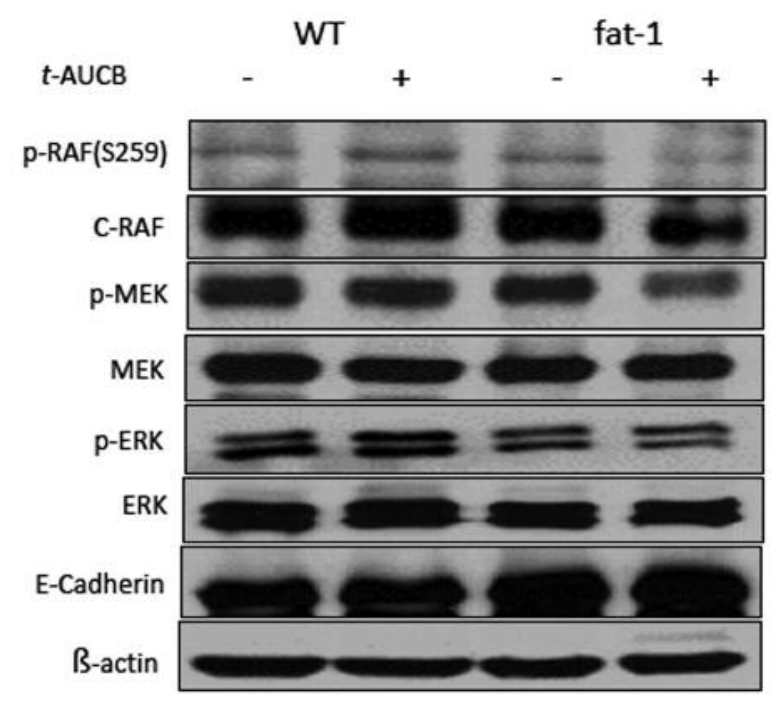

Figure 3. Western blot assay for freshly collected PKO3 tumors developing from wild-type (WT) and fat-1 transgenic mice for activated (phospho, $p$-) and inactive KRAS downstream signals of phosphorylated serine/threonine kinases (cRAF, S259), mitogen-activated protein kinase kinase $(M E K)$, extracellular signal-regulated kinase (ERK) and $E$-cadherin ( $\beta$-actin was used as a protein loading control).

acids, we particularly focused on epoxide metabolites by analyzing the ratios of epoxyeicosatrienoic acids (EETs)/ dihydroxyeicosatrienoic acids (DHETs) and epoxyoctadecaenoic acids (EpOMEs)/dihydroxyoctade-caenoic acids (DiHOMEs) as biomarkers for determining the effects of $\mathrm{sEH}$ inhibition/knockout mediated epoxy fatty acid metabolism. As seen in Figure 4A and B, increased ratios of EpOMEs/DiHOMs for majority of the $\omega-3$ PUFAs, including EPA and DHA, were observed in fat- 1 transgenic mice compared to those in C57BL/6J mice. However, changes the ratios of EETs/DHETs and EpOMEs/DiHOMs for the majority of $\omega-6$ PUFAs, including LA and arachidonic acid, were not significant, except for linoleic acid and its 9,10 epoxide (Figure $4 \mathrm{C}$ and $\mathrm{D}$ ).

For analyzing the effect of sEH inhibition or gene knockout on modulating cyclooxygenase (COX2) and lipoxygenase (LOX)-mediated metabolic pathways of $\omega-6$ fatty acids, the levels of PGE2 (prostaglandin E2), PGD2 (prostaglandin D2), TXB2 (thromboxane B2), LTB4 (Leukotriene B4) and 5-HETE (5-hydroxyeicosatetraenoic acid) were analyzed. Our results showed that there were no significant differences among these treated mice (data not shown).

\section{Discussion}

Recent studies pointed out the potential role of $\omega-3$ epoxy fatty acid metabolites in $\omega-3$ fatty acid-/fish oil-based cancer prevention and therapy; in particular, the synthetic analog of $\omega$-3 17,18-epoxyeicosatetraenoic acid efficiently inhibited intra-mammary cancer cell xenografts in mice and promoted apoptosis of human breast cancer cells $(35,36)$, and esophageal squamous carcinoma (37). Since $\omega-3$ epoxides are the dominant metabolites of $\omega-3$ PUFAs, in the present study, we further analyzed the effect of $\omega$-3 epoxy fatty acids on pancreatic cancer growth using a murine PK03 PDAC cancer model. Our results demonstrated significant inhibition of PK03 PDAC cancer growth in C57BL/6J mice fed an $\omega-$ 3 -rich fish oil diet and treated with sEH inhibitor; in $\mathrm{sEH}$ knockout mice fed an $\omega$-3-rich fish oil diet; and in fat-1 transgenic mice treated with $\mathrm{sEH}$ inhibitor.

The fat-1 transgenic mouse is an excellent model for studying the biology of $\omega-3$ fatty acids, particularly the transgenic fatty acid desaturase which converts endogenous $\omega-6$ to $\omega-3$ fatty acids (21). Lipidomic profiling revealed that sEH inhibition/knockout lead to accumulation/stabilization of $\omega-3$ epoxides. In particular, we demonstrated that the ratio of EpOME/DiHOM derived from EPA and DHA was significantly increased in fat- 1 transgenic mice treated with $t$-AUCB, but not from $\omega-6$ fatty acids (including arachidonic acid and Linoleic acid) compared to the C57BL/6J mice. These results indicate $\omega-3$ epoxy metabolites is an important player in biological function of $\omega-3$ fatty acids.

Active inflammatory cells (including neutrophils and macrophages) not only are the crucial players in the inflammatory process, but also promote malignant progression from its initiation, and growth to metastasis. Neutrophils, the terminally differentiated effector in active inflammation, play a major role in the acute phase of inflammation and against microbes, mainly via producing cytokines and effector molecules, and interacting with distinct cell populations. Most importantly, neutrophils are involved in the regulation of both innate and adaptive immune responses in the inflammatory process and cancer. MPO is the major component of neutrophil azurophilic granules, which is responsible for active neutrophil-related tissue damage. MPO also serves as a relatively specific biomarker for detecting neutrophils. We further identified a significant decrease of MPO-labeled active neutrophils in the PKO3 tumors in the fat- 1 transgenic mice and C57BL/6J mice fed with fish oil diet combined with sEH inhibition or knockout compared to the C57BL/6J mice fed with AIN-76A diet, supporting the role of $\omega-3$ epoxy metabolites against acute inflammatory cells-neutrophils infiltration.

Angiogenesis is an essential step for tumor growth and metastasis. A potential pro-angiogenic effect of $\mathrm{sEH}$ inhibition has been noted, particularly through $\omega-6$ epoxy metabolitemediated angiogenesis that can potentially promote tumor progression or metastasis $(38,39)$. However, $\omega-3$ epoxy metabolites have an opposite effect, inhibiting angiogenesis and blocking tumor growth and metastasis (7). Our results demonstrated CD34 labeled MVD was significantly lower in 

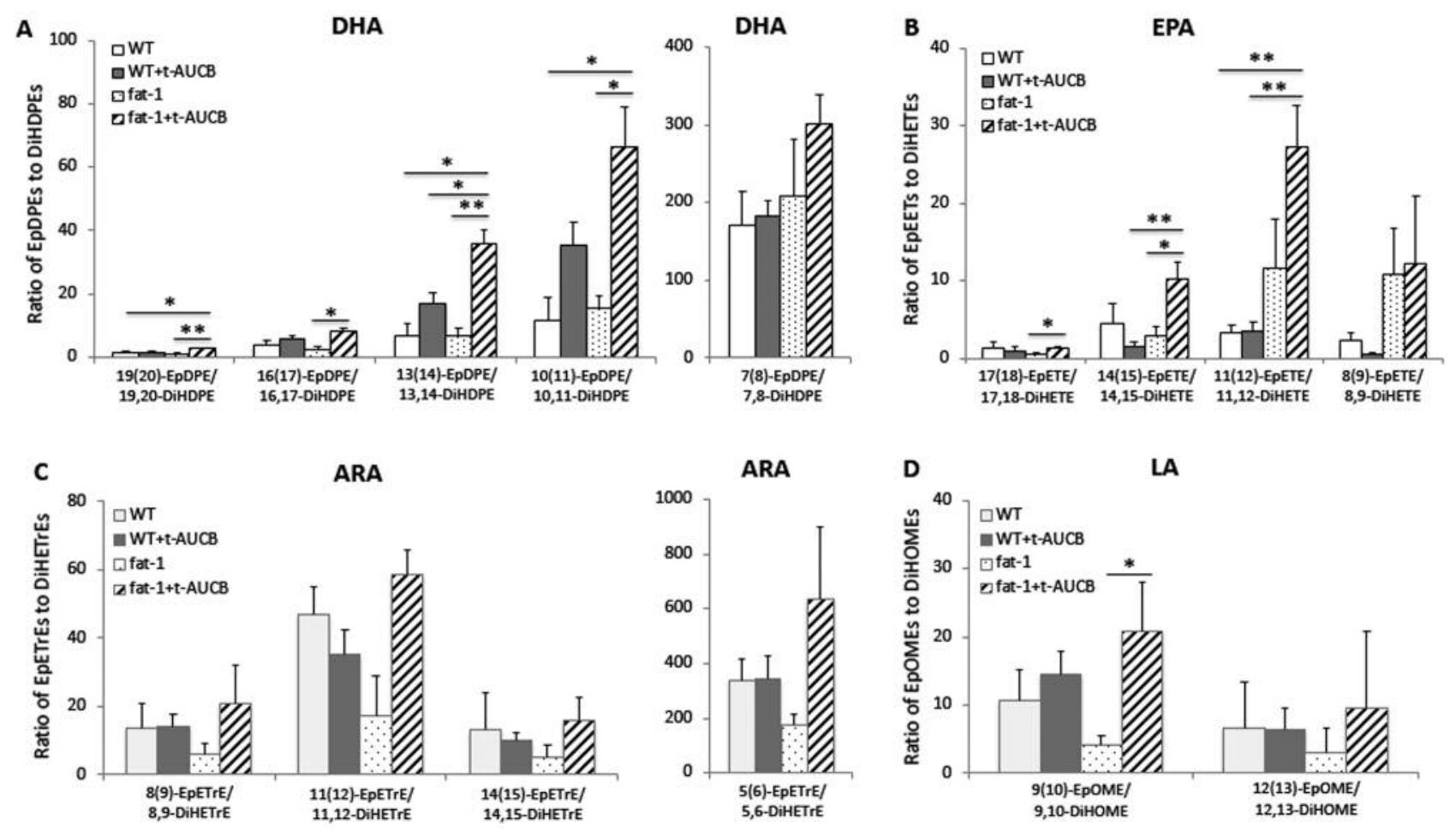

Figure 4. Polyunsaturated fatty acid (PUFA) profile in plasma specimens analyzed using liquid chromatography with tandem mass spectrometry. The ratio of epoxides to diols as the best biomarker for soluble epoxide hydrolase (Ephx2) knockout (sEH $H^{--}$) and inhibition was analyzed. The significant metabolites of epoxides and diols analyzed were epoxydocosapentaenoic acids (EpDPEs) and dihydroxydocosapentaenoic acids (DiHDPEs) from docosahexaenoic acid (DHA) (A), epoxyeicosatetraenoic acids (EpETEs) and dihydroxyeicosatetraenoic acids (DiHETEs) from eicosapentaenoic acid (EPA) (B), epoxyeicosatrienoic acids (EPETrEs) and dihydroxyeicosatrienoic acids (DiHETrEs) from arachidonic acid (ARA) $(C)$, and epoxyoctadecamonoenoic acids (EpOMEs) and dihydroxyoctadecenoic acids (DiHOMEs) (D) from linoleic acid (LA).

fat-1 transgenic mice combined with sEH inhibition compared to the C57BL/6J mice, further confirming the role of $\omega-3$ epoxy lipid metabolites in inhibiting angiogenesis.

The RAS/RAF/MEK/ERK cascade plays a crucial role in the regulation of cell cycle/proliferation and apoptosis. Our results showed that the phosphorylation of c-RAF, MEK $1 / 2$ and ERK1/2 (mutant KRAS-activated signals) were markedly reduced in the PK03 PDAC cell tumors in fat- 1 transgenic mice treated with $t$-AUCB compared to $\mathrm{C} 57 \mathrm{BL} / 6 \mathrm{~J}$ mice treated with/without $t$-AUCB and fat-1 transgenic mice treated without $t$-AUCB. Moreover, increased expression of E-cadherin was also found in the PK03 tumors from fat- 1 transgenic mice compared to C57BL/6J mice. These data indicate that $\omega-3$ epoxy metabolites play an important role in inhibiting growth of PDAC via inhibiting the RAS/RAF/ MEK/ERK pathway.

In summary, to the best of our knowledge, this is the first report that identified the inhibitory role of $\omega$ - 3 epoxides in the growth of pancreatic ductal adenocarcinoma. Our results indicate that $\omega-3$ epoxy metabolites play an important role in inhibiting pancreatic carcinogenesis and use of $\omega-3$ PUFAs together with sEH inhibition may be a highly effective approach for inhibiting pancreatic cancer.

\section{Conflicts of Interest}

The Authors declare no conflicts of interest.

\section{Authors' Contributions}

RX, LS, JL and GYY planned the study. RX, LS, JL HL, XY, DX, J Y, $\mathrm{SHH}, \mathrm{RDJ}, \mathrm{BH}$ and GYY performed the experiments, analyzed the data and prepared figures. BH and GYY provided guidance to the study and corrected the manuscript. All Authors reviewed the manuscript.

\section{Acknowledgements}

This study was supported by NIH R01 DK10776, CA172431, and CA164041 to Dr. Guang-Yu Yang.

\section{References}

1 Fischer R, Konkel A, Mehling H, Blossey K, Gapelyuk A, Wessel N, von Schacky C, Dechend R, Muller DN, Rothe M, Luft FC, Weylandt K and Schunck WH: Dietary omega-3 fatty acids modulate the eicosanoid profile in man primarily via the cyp-epoxygenase pathway. J Lipid Res 55(6): 1150-1164, 2014. PMID: 24634501. DOI: 10.1194/jlr.M047357 
2 Astarita G, McKenzie JH, Wang B, Strassburg K, Doneanu A, Johnson J, Baker A, Hankemeier T, Murphy J, Vreeken RJ, Langridge $\mathrm{J}$ and Kang JX: A protective lipidomic biosignature associated with a balanced omega-6/omega- 3 ratio in fat- 1 transgenic mice. PLoS One 9(4): e96221, 2014. PMID: 24760204. DOI: 10.1371/journal.pone.0096221

3 Arnold C, Markovic M, Blossey K, Wallukat G, Fischer R, Dechend R, Konkel A, von Schacky C, Luft FC, Muller DN, Rothe $\mathrm{M}$ and Schunck WH: Arachidonic acid-metabolizing cytochrome p450 enzymes are targets of \{omega\}-3 fatty acids. J Biol Chem 285(43): 32720-32733, 2010. PMID: 20732876. DOI: $10.1074 / j b c . M 110.118406$

4 Jump DB: The biochemistry of n-3 polyunsaturated fatty acids. J Biol Chem 277(11): 8755-8758, 2002. PMID: 11748246. DOI: $10.1074 /$ jbc.R100062200

5 Malkowski MG, Thuresson ED, Lakkides KM, Rieke CJ, Micielli R, Smith WL and Garavito RM: Structure of eicosapentaenoic and linoleic acids in the cyclooxygenase site of prostaglandin endoperoxide h synthase-1. J Biol Chem 276(40): 37547-37555, 2001. PMID: 11477109 . DOI: $10.1074 /$ jbc.M105982200

6 Laneuville O, Breuer DK, Xu N, Huang ZH, Gage DA, Watson JT, Lagarde M, DeWitt DL and Smith WL: Fatty acid substrate specificities of human prostaglandin-endoperoxide $h$ synthase- 1 and -2 . Formation of 12-hydroxy-(9z, 13e/z, 15z)- octadecatrienoic acids from alpha-linolenic acid. J Biol Chem 270(33): 1933019336, 1995. PMID: 7642610. DOI: 10.1074/ jbc.270.33.19330

7 Zhang G, Panigrahy D, Mahakian LM, Yang J, Liu JY, Stephen Lee KS, Wettersten HI, Ulu A, Hu X, Tam S, Hwang SH, Ingham ES, Kieran MW, Weiss RH, Ferrara KW and Hammock BD: Epoxy metabolites of docosahexaenoic acid (dha) inhibit angiogenesis, tumor growth, and metastasis. Proc Natl Acad Sci USA 110(16): 6530-6535, 2013. PMID: 23553837. DOI: 10.1073/pnas.1304321110

8 Morin C, Sirois M, Echave V, Albadine R and Rousseau E: 17,18-epoxyeicosatetraenoic acid targets ppargamma and $\mathrm{p} 38$ mitogen-activated protein kinase to mediate its antiinflammatory effects in the lung: Role of soluble epoxide hydrolase. Am J Respir Cell Mol Biol 43(5): 564-575, 2010. PMID: 20008283. DOI: $10.1165 / \mathrm{rcmb} .2009-01550 C$

9 Kubota T, Arita M, Isobe Y, Iwamoto R, Goto T, Yoshioka T, Urabe D, Inoue $\mathrm{M}$ and Arai $\mathrm{H}$ : Eicosapentaenoic acid is converted via omega-3 epoxygenation to the anti-inflammatory metabolite 12-hydroxy-17,18-epoxyeicosatetraenoic acid. FASEB J 28(2): 586-593, 2014. PMID: 24128889. DOI: 10.1096/fj.13-236224

10 Cui PH, Petrovic N and Murray M: The omega-3 epoxide of eicosapentaenoic acid inhibits endothelial cell proliferation by p38 map kinase activation and cyclin d1/cdk4 down-regulation. Br J Pharmacol 162(5): 1143-1155, 2011. PMID: 21077851. DOI: $10.1111 / \mathrm{j} .1476-5381.2010 .01113 . \mathrm{x}$

11 Norwood S, Liao J, Hammock BD and Yang GY: Epoxyeicosatrienoic acids and soluble epoxide hydrolase: Potential therapeutic targets for inflammation and its induced carcinogenesis. Am J Transl Res 2(4): 447-457, 2010. PMID: 20733953.

12 Ulu A, Harris TR, Morisseau C, Miyabe C, Inoue H, Schuster G, Dong H, Iosif AM, Liu JY, Weiss RH, Chiamvimonvat N, Imig JD and Hammock BD: Anti-inflammatory effects of omega-3 polyunsaturated fatty acids and soluble epoxide hydrolase inhibitors in angiotensin-ii-dependent hypertension. J Cardiovasc Pharmacol 62(3): 285-297, 2013. PMID: 23676336. DOI: $10.1097 /$ FJC.0b013e318298e 460
13 Zhang G, Kodani S and Hammock BD: Stabilized epoxygenated fatty acids regulate inflammation, pain, angiogenesis and cancer. Prog Lipid Res 53: 108-123, 2014. PMID: 24345640. DOI: 10.1016/j.plipres.2013.11.003

14 Lopez-Vicario C, Alcaraz-Quiles J, Garcia-Alonso V, Rius B, Hwang SH, Titos E, Lopategi A, Hammock BD, Arroyo V and Claria J: Inhibition of soluble epoxide hydrolase modulates inflammation and autophagy in obese adipose tissue and liver: Role for omega-3 epoxides. Proc Natl Acad Sci USA 112(2): 536-541, 2015. PMID: 25550510. DOI: 10.1073/pnas.1422 590112

$15 \mathrm{Xu}$ DY, Davis BB, Wang ZH, Zhao SP, Wasti B, Liu ZL, Li N, Morisseau C, Chiamvimonvat $\mathrm{N}$ and Hammock BD: A potent soluble epoxide hydrolase inhibitor, t-aucb, acts through ppargamma to modulate the function of endothelial progenitor cells from patients with acute myocardial infarction. Int J Cardiol 167(4): 1298-1304, 2013. PMID: 22525341. DOI: 10.1016/j.ijcard.2012.03.167

16 Hwang SH, Wecksler AT, Zhang G, Morisseau C, Nguyen LV, Fu SH and Hammock BD: Synthesis and biological evaluation of sorafenib- and regorafenib-like seh inhibitors. Bioorg Med Chem Lett 23(13): 3732-3737, 2013. PMID: 23726028. DOI: 10.1016/j.bmcl.2013.05.011

17 Zhang W, Li H, Dong H, Liao J, Hammock BD and Yang GY: Soluble epoxide hydrolase deficiency inhibits dextran sulfate sodium-induced colitis and carcinogenesis in mice. Anticancer Res 33(12): 5261-5271, 2013. PMID: 24324059.

18 Funahashi H, Satake M, Hasan S, Sawai H, Newman RA, Reber HA, Hines OJ and Eibl G: Opposing effects of n-6 and n-3 polyunsaturated fatty acids on pancreatic cancer growth. Pancreas 36(4): 353-362, 2008. PMID: 18437081. DOI: 10.1097/MPA.0b013e31815ccc44

19 Williams CD, Whitley BM, Hoyo C, Grant DJ, Iraggi JD, Newman KA, Gerber L, Taylor LA, McKeever MG and Freedland SJ: A high ratio of dietary n-6/n-3 polyunsaturated fatty acids is associated with increased risk of prostate cancer. Nutr Res 31(1): 1-8, 2011. PMID: 21310299. DOI: 10.1016/ j.nutres.2011.01.002

20 Liu HQ, Qiu Y, Mu Y, Zhang XJ, Liu L, Hou XH, Zhang L, Xu $\mathrm{XN}$, Ji AL, Cao R, Yang RH and Wang F: A high ratio of dietary n-3/n-6 polyunsaturated fatty acids improves obesity-linked inflammation and insulin resistance through suppressing activation of tlr4 in sd rats. Nutr Res 33(10): 849-858, 2013. PMID: 24074743. DOI: 10.1016/j.nutres.2013.07.004

21 Kang JX: Fat-1 transgenic mice: A new model for omega-3 research. Prostaglandins Leukot Essent Fatty Acids 77(5-6): 263267, 2007. PMID: 18042365. DOI: 10.1016/j.plefa.2007.10.010

22 Siegel RL, Miller KD and Jemal A: Cancer statistics, 2018. CA Cancer J Clin 68(1): 7-30, 2018. PMID: 29313949. DOI: $10.3322 / \mathrm{caac} .21442$

$23 \mathrm{Li} \mathrm{H}$, Yang AL, Chung YT, Zhang W, Liao J and Yang GY: Sulindac inhibits pancreatic carcinogenesis in lsl-krasg12d-lsltrp53r172h-pdx-1-cre mice via suppressing aldo-keto reductase family 1b10 (akr1b10). Carcinogenesis 34(9): 2090-2098, 2013. PMID: 23689354. DOI: 10.1093/carcin/bgt170

24 Liao J, Chung YT, Yang AL, Zhang M, Li H, Zhang W, Yan L and Yang GY: Atorvastatin inhibits pancreatic carcinogenesis and increases survival in 1sl-kras(g12d) -lsl-trp53(r172h) -pdx1cre mice. Mol Carcinog, 2012. PMID: 22549877. DOI: 10.1002/ mc. 21916 
25 Souza RF, Lei J, Yin J, Appel R, Zou T-T, Zhou X, Wang S, Rhyu M-G, Cymes K, Chan O, Park W-S, Krasna MJ, Greenwald BD, Cottrell J, Abraham JM, Simms L, Leggett B, Young J, Harpaz N and Meltzer SJ: A transforming growth factor b1 receptor type ii mutation in ulcerative colitis-associated neoplasms. Gastroenterology 112: 40-45, 1997. PMID: 8978341

26 Ryan DP, Hong TS and Bardeesy N: Pancreatic adenocarcinoma. N Engl J Med 371(22): 2140-2141, 2014. PMID: 25207767. DOI: $10.1056 / \mathrm{NEJMra1404198}$

27 Pylayeva-Gupta Y, Grabocka E and Bar-Sagi D: Ras oncogenes: Weaving a tumorigenic web. Nat Rev Cancer 11(11): 761-774, 2011. PMID: 21993244. DOI: $10.1038 / \mathrm{nrc} 3106$

28 Berra E, Diaz-Meco MT and Moscat J: The activation of p38 and apoptosis by the inhibition of erk is antagonized by the phosphoinositide 3-kinase/akt pathway. J Biol Chem 273(17): 10792-10797, 1998. PMID: 9553146. DOI: 10.1074/ jbc.273.17.10792

29 Bai H, Li H, Zhang W, Matkowskyj KA, Liao J, Srivastava SK and Yang GY: Inhibition of chronic pancreatitis and pancreatic intraepithelial neoplasia (panin) by capsaicin in lslkrasg12d/pdx1-cre mice. Carcinogenesis 32(11): 1689-1696, 2011. PMID: 21859833. DOI: 10.1093/carcin/bgr191

30 Lau BY, Ward WE, Kang JX and Ma DW: Fat-1 gene modulates the fatty acid composition of femoral and vertebral phospholipids. Appl Physiol Nutr Metab 35(4): 447-455, 2010. PMID: 20725110. DOI: 10.1139/H10-029

31 Zhang W, Li H, Yang Y, Liao J and Yang GY: Knockdown or inhibition of aldo-keto reductase $1 \mathrm{~b} 10$ inhibits pancreatic carcinoma growth via modulating kras-e-cadherin pathway. Cancer Lett 355(2): 273-280, 2014. PMID: 25304374. DOI: 10.1016/j.canlet.2014.09.031

32 Liao J, Hwang SH, Li H, Yang Y, Yang J, Wecksler AT, Liu JY, Hammock BD and Yang GY: Inhibition of mutant krasg12dinitiated murine pancreatic carcinoma growth by a dual c-raf and soluble epoxide hydrolase inhibitor t-cupm. Cancer Lett 371(2): 187-193, 2016. PMID: 26683769. DOI: 10.1016/j.canlet. 2015.11.042

33 Liao J, Hwang SH, Li H, Liu JY, Hammock BD and Yang GY: Inhibition of chronic pancreatitis and murine pancreatic intraepithelial neoplasia by a dual inhibitor of c-raf and soluble epoxide hydrolase in 1sl-krasg(1)(2)d/pdx-1-cre mice. Anticancer Res 36(1): 27-37, 2016. PMID: 26722025.
34 Zhang W, Yang AL, Liao J, Li H, Dong H, Chung YT, Bai H, Matkowskyj KA, Hammock BD and Yang GY: Soluble epoxide hydrolase gene deficiency or inhibition attenuates chronic active inflammatory bowel disease in il-10(-/-) mice. Dig Dis Sci 57(10): 2580-2591, 2012. PMID: 22588244. DOI: 10.1007/ s10620-012-2217-1

35 Dyari HRE, Rawling T, Chen Y, Sudarmana W, Bourget K, Dwyer JM, Allison SE and Murray M: A novel synthetic analogue of omega-3 17,18-epoxyeicosatetraenoic acid activates tnf receptor-1/ask1/jnk signaling to promote apoptosis in human breast cancer cells. FASEB J 31(12): 5246-5257, 2017. PMID: 28798154. DOI: 10.1096/fj.201700033R

36 Dyari HR, Rawling T, Bourget K and Murray M: Synthetic omega-3 epoxyfatty acids as antiproliferative and pro-apoptotic agents in human breast cancer cells. J Med Chem 57(17): 74597464, 2014. PMID: 25144895. DOI: 10.1021/jm501083y

37 Mizoguchi K, Ishiguro H, Kimura M, Takahashi H, Sakamoto $\mathrm{N}$, Tanaka $\mathrm{T}$ and Takeyama $\mathrm{H}$ : Induction of apoptosis by eicosapentaenoic acid in esophageal squamous cell carcinoma. Anticancer Res 34(12): 7145-7149, 2014. PMID: 25503142.

38 Elson $\mathrm{CE}$ and Yu SG: The chemoprevention of cancer by mevalonate-derived constituents of fruits and vegetables. J Nutr 124(5): 607-614, 1994. PMID: 8169651. DOI: 10.1093/jn/ 124.5.607

39 Panigrahy D, Edin ML, Lee CR, Huang S, Bielenberg DR, Butterfield CE, Barnes CM, Mammoto A, Mammoto T, Luria A, Benny O, Chaponis DM, Dudley AC, Greene ER, Vergilio JA, Pietramaggiori G, Scherer-Pietramaggiori SS, Short SM, Seth M, Lih FB, Tomer KB, Yang J, Schwendener RA, Hammock BD, Falck JR, Manthati VL, Ingber DE, Kaipainen A, D'Amore PA, Kieran MW and Zeldin DC: Epoxyeicosanoids stimulate multiorgan metastasis and tumor dormancy escape in mice. $\mathrm{J}$ Clin Invest 122(1): 178-191, 2012. PMID: 22182838. DOI: $10.1172 /$ JCI58128
Received March 15, 2019

Revised May 30, 2019

Accepted June 10, 2019 